\title{
Positioning Trocars for Performing Sleeve Gastrectomy: the right side or between the legs of the patient?
}

\author{
Tarek Debs $^{1}$ - Imed Ben Amor ${ }^{1}$. Jean Gugenheim ${ }^{1}$ • Radwan Kassir ${ }^{2}$
}

Published online: 24 July 2015

(C) Springer Science+Business Media New York 2015

Dear Editor,

We agree that the number of trocars is variable in performing sleeve gastrectomy [1]. In the same time, we do not agree with Ruiz de Angulo et al. [2], and we think that the location of the trocars remains also variable as it depends on the habits of the surgeon and the position of the operator, whether on the right side or between the legs of the patient [3].

In a standard sleeve gastrectomy, the whole body of the stomach and the proximal part of the antrum should be completely freed along the greater curvature. This mobilization will allow an easy manipulation of the stomach. In some centers where the operator stands on the right side of the patient, the stapling during the sleeve gastrectomy is performed through the trocar on the right side of the patient. However, if the surgeon stands between the legs of the patient, the stapling could be performed either through the trocar on the right side of the patient, or through the trocar on the left side of the patient. The mobilization of the stomach allows an easy manipulation and therefore the placement of the first stapling through either side without closing the angle at the incisura angularis and risking a stenosis.

Radwan Kassir

Radwankassir42@hotmail.Fr

Department of Bariatric Surgery, Hospital Archet 2, Nice, France

2 Department of General Surgery, CHU Hospital, Jean Monnet University, Avenue Albert Raimond, 42270 Saint Etienne, France
Another important aspect in laparoscopic surgery that facilitates the use of the stapler through either side is the development of the stapling devices. They are designed to be easy to use and to facilitate tissue positioning and manipulation with their wide proximal-to-distal jaw aperture. The articulation allows the surgeon to focus on the line of transection and place the anvil exactly where needed. With its natural articulation, the shaft can rotate freely in both directions. An articulation mechanism enables the distal portion of the shaft to pivot to facilitate lateral access to the operative site [4].

Declaration of Interest The authors declare that they have no competing interests.

Statement of Informed Consent Informed consent was obtained from all individual participants included in the study.

Statement of Human and Animal Rights Informed consent was obtained from all individual participants included in the study.

\section{References}

1. Kassir R, Lointier P, Breton C, et al. Positioning trocars for performing sleeve gastrectomy. Points of Controversy Obes Surg. 2015;25(4):712.

2. Ruiz de Angulo D, Munitiz V, Ortiz MÁ, et al. Positioning trocars for performing sleeve gastrectomy. Points of controversy. Obes Surg. 2015;25(5):854.

3. Gentileschi P, Camperchioli I, Benavoli D, et al. Laparoscopic single-port sleeve gastrectomy for morbid obesity: preliminary series. Surg Obes Relat Dis. 2010;6(6):665-9.

4. Fuchs KH, Breithaupt W, Schulz T, et al. Experience with flexible stapling techniques in laparoscopic and conventional surgery. Surg Endosc. 2011;25(6):1783-90. 